\section{$\underset{\substack{\text { hommes } \\ \text { \& migrations }}}{ }$}

\section{Hommes \& migrations}

Revue française de référence sur les dynamiques

migratoires

1303 | 2013

Diasporas marocaines

\title{
Alimentation et identité entre deux rives
}

\author{
Marie-Pierre Étien et Laurence Tibère
}

URL : http://journals.openedition.org/hommesmigrations/2552

DOI : 10.4000/hommesmigrations.2552

ISSN : 2262-3353

\section{Éditeur}

Musée national de l'histoire de l'immigration

\section{Édition imprimée}

Date de publication : 1 juillet 2013

Pagination : 57-64

ISBN : 978-2-919040-23-0

ISSN : $1142-852 X$

\section{Référence électronique}

Marie-Pierre Étien et Laurence Tibère, «Alimentation et identité entre deux rives », Hommes \& 


\section{ALIMENTATION ET IDENTITÉ ENTRE DEUX RIVES}

par MARIE-PIERRE ÉTIEN, doctorante en sociologie,Certop (UMR-CNRS 5044), Université Toulouse-II Le Mirail (France), et LAURENCE TIBÈRE, maître de conférences en sociologie, Certop (UMR-CNRS 5044), Université Toulouse-II Le Mirail (France), Taylors University, Chair of food studies (Malaisie)

Que l'on soit né au Maroc ou en France de parents marocains, I'alimentation occupe une place centrale dans le tissage du lien avec ses origines réelles, rêvées ou mythiques. À travers l'analyse de récits de vie de Marocains vivant en France, il s'agit de comprendre comment l'alimentation nourrit les constructions identitaires, individuelles et collectives, comment elle intervient dans la construction de sentiments d'appartenance et d'intégration, et comment elle permet à l'esprit et au corps de s'amarrer à un ici ou à un ailleurs.

\section{Pratiques alimentaires et (re)compositions identitaires des Marocains vivant en France}

L'acte alimentaire est porteur de sens. En mangeant certains plats ou en les refusant, on exprime, plus ou moins consciemment, qui l'on est, qui l'on veut être ou qui l'on ne veut pas être. On raconte son histoire et on prend place dans la société dans laquelle on vit. L'alimentation sert la création identitaire autant que la langue ou la musique ${ }^{1}$. Entendue comme un des systèmes symboliques mobilisés pour produire du sens, elle intervient dans les constructions sociales autour de la mémoire et du "positionnement identitaire". Comment l'alimentation agit- elle dans le parcours migratoire et l'installation dans une nouvelle société ? Quel est son statut dans les perceptions des populations issues de l'immigration ? Comment s'opèrent les constructions matérielles et symboliques qui accompagnent l'expérience migratoire?

Pour analyser le lien entre l'alimentation, la mémoire, l'identité et la construction des origines, nous avons entendu ${ }^{3}$ une trentaine d'hommes et de femmes marocains ou d'origine marocaine âgés de 21 à 65 ans sur leur histoire familiale et 
aussi appeler "sémiosphère du manger", puis des souvenirs liés aux odeurs et aux goûts ${ }^{5}$. Les sens et les perceptions ont été privilégiés car dans l'exposition identitaire l'important n'est pas le vrai, l'objectif, le réel, mais la construction des sens ${ }^{6}$. Cet article propose d'analyser les processus de socialisation et de resocialisation alimentaires postérieurs à la migration et la façon dont ils s'adossent à des repositionnements identitaires. Nous nous focaliserons d'abord sur le cas de Marocains nés et ayant eu une socialisation alimentaire au Maroc. Puis nous nous concentrerons sur le cas de Français d'origine marocaine mais pas seulement, car il s'agira de comprendre comment l'alimentation est à la fois liée aux relations entretenues avec la société d'origine et la société dans laquelle on vit, et comment les jeux identitaires qui en découlent contribuent à se sentir d'ici, de France et/ou d'ailleurs, du Maroc.

Youssef Mansouri, un Marocain de 26 ans arrivé en France pour ses études et faisant l'objet d'un arrêté d'expulsion en 2006 tient une pizzeria en arrière plan et y emploie quatre personnes $\odot$ AfP Photo Franck Perry

personnelle, sur le parcours migratoire vécu ou son souvenir raconté par les parents, l'enfance et la scolarité, l'entrée dans le monde du travail, la vie d'aujourd'hui et les projets pour demain. Nous sommes parties du récit de vie des individus en repérant comment l'alimentation était mobilisée. Pour comprendre le statut symbolique de l'alimentation dans les parcours migratoires et capter des identités en mouvement, nous nous sommes appliquées à recueillir des ressentis plus que des faits concrets. Du sens et des sens : des univers de signification liés à l'alimentation que l'on peut
Qu'ils aient traversé la Méditerranée dans les années 1970 ou plus récemment dans les années 2000, nous repérons chez tous les interviewés nés au Maroc et arrivés en France entre l'âge de 18 et 21 ans une resocialisation alimentaire en trois temps. Il s'agit d'un processus révélant un parcours alimentaire post-migratoire en trois phases. La première est une phase de perturbation identitaire touchant les principales dimensions de l'“espace social alimentaire ${ }^{7 ”}$. Durant cette étape, les Marocains rencontrés évoquent leurs difficultés à faire face à ce qu'ils vivent comme une baisse qualitative de leur alimentation, mais en même temps certaines habitudes persistent. Les représentations de partage, de convivialité et de lien social 
autour de la nourriture, tout comme la symbolique des aliments et les modalités de consommation, se trouvent bouleversées et font l'objet de diverses adaptations compensatoires. Cela ne signifie pas la perte de la culture d'origine. On sait que, selon les contextes, les pratiques alimentaires "oscillent entre continuité/discontinuité et discontinuité/continuitée". Les mécanismes porteurs de métissage s'inscrivent dans ces bricolages et restent bien souvent adossés à un "manger marocain" cristallisé sur des composantes "totems" : kebab, BMK (bide/matecha/ kefta, correspondant à un tajine d'œuf, tomate et viande hachée), pain associé à de l'huile d'olive, miel, thé et conserves en tout genre agrémentées d'épices. L'alimentation marocaine est oubliée un moment, soit par l'indisponibilité des ressources alimentaires, soit par manque de savoir-faire culinaire, soit par désir d'ouverture et d'intégration.

La seconde phase repose sur des (re)compositions identitaires naviguant entre un "manger d'ici" et un "manger de là-bas". Les normes comme les symboles se mêlent. Les interviewés passent du manger marocain au manger français ou du "français à la marocaine" et vice versa. Ils mangent français certains jours de la semaine, souvent par commodité, et marocain d'autres jours lorsqu'ils ont plus de temps. Il y a des jours avec, des jours sans, mais des épices tout le temps. Parfois, le manger marocain reste très exceptionnel et marque une certaine temporalité se rattachant à leurs origines. Ils mangent marocain pour les mariages, les naissances, les enterrements et toujours pendant le ramadan pour faire entrer le Maroc en soi pendant un mois: "Je mange très rarement marocain. Ce qui est sûr, c'est que ma dose de nourriture marocaine, je la prends pendant le ramadan! Je me remplis pour l'année et ça me suffit" (Mustafa, 39 ans). Dans certaines familles, le couscous du vendredi se décale au dimanche pour avoir tout le monde autour de la table : "Le couscous, maintenant, je le fais le dimanche quand on est tous réunis" (Lasisa, 61 ans). Enfin, la troisième et dernière phase du cycle par lequel passent toutes les personnes rencontrées révèle une appropriation et la cohabitation plus ou moins harmonieuse, très souvent argumentée et justifiée a posteriori, de différents codes du manger français et du manger marocain avec lesquels ils jouent pour se positionner en fonction de contextes d'interaction. Ce qui est important ici, ce n'est pas la cohabitation des modèles alimentaires, déjà présente au début du processus, mais les jeux identitaires et la mise en scène de $s{ }^{10}{ }^{10}$ qu'ils manifestent. Ces jeux se révèlent surtout lorsque l'on reçoit chez soi et pointent aussi l'importance de l'utilisation des nouvelles technologies dans les apprentissages, les partages et la transmission des savoir-faire culinaires. Ils téléphonent aux parents restés au pays pour avoir les recettes, ils regardent sur le Net ou se laissent attraper par Choumicha à la télévision. Cuisiner un plat de tradition familiale devient alors une façon d'exprimer aux autres ${ }^{11}$ cette Dans certaines familles, le couscous du vendredi se décale au dimanche part de soi et de son identité dont on ne pourra jamais pour avoir tout le monde autour de la table. se défaire. Désir d'identification et d'appartenance, mais aussi expression des spécificités, sont au cœur des stratégies qui poussent à exprimer sa singularité tout en prenant position au sein d'un groupe. Nous nommons ce processus construction d'une "personnalité alimentaire"12. À l'opposé, certains interviewés choisissent de mettre en scène l'adoption de certains codes de la culture alimentaire française comme l'individualisation de la vaisselle. Autant de symboles pour eux et leur famille de leur identité française ou en tout cas de ce qu'ils considèrent comme représentatif de l'univers français. Ce jeu entre univers marocain et univers français, qui est à la fois autre et proche par réappropriation, se ren- 
contre souvent ${ }^{13}$. La différence est alors vécue comme un atout, un élément à valoriser et à dévoiler aux autres en même temps qu'on se dévoile soi-même. Plusieurs enquêtés déclarent adorer cuisiner des tajines, des couscous et autres soupes pour leurs amis, histoire de leur faire découvrir leur cuisine... ou qui ils sont au fond. Étudier la façon dont ces populations prennent place au sein de la société d'accueil par l'alimentation tout en maintenant ou non le fil avec

La question des modalités

d'accès à certains produits alimentaires est déterminante dans l'évolution des modèles

alimentaires et se lie aux phénomènes d'acculturation et d'implantation

dans la société d'accueil. le pays des origines ${ }^{14}$ permet de repenser les (re)socialisationsalimentaires en les inscrivant dans un processus se déroulant finalement tout au long de la vie et pour tout le monde. $\mathrm{Ce}$ qu'il faut garder à l'esprit, c'est que tous les interviewés passent par ces phases, mais chacun les vit selon des intensités différentes qui dépendent en grande partie des contextes de la migration et des dimensions politiques, économiques, socioculturelles ou matérielles. La question des modalités d'accès à certains produits alimentaires est par exemple déterminante dans l'évolution des modèles alimentaires et se lie aux phénomènes d'acculturation et d'implantation dans la société d'accueil. La provenance géographique, le milieu social de l'individu, son niveau d'éducation ou encore son degré de religiosité ont aussi leur importance.

\section{L'expérience d'une dualité}

Pour les enquêtés nés en France de parents marocains, la socialisation alimentaire est portée par une double dynamique. Il y a d'une part ce qui est présenté comme produit par la mémoire familiale, avec l'idée d'une transmission d'un manger maro- cain plus ou moins accepté et revendiqué selon les cas et, d'autre part, ce qui se construit, s'élabore au présent et dans l'ici, dans la société majoritaire. Cette dualité va sans cesse être en mouvement en fonction d'aspects tels que les contextes et formes d'interactions, les âges ou encore les préférences alimentaires. L'exposition d'un manger coincé entre deux rives se retrouve principalement chez les personnes se déclarant "Françaises d'origine marocaine", mais pas seulement, car il est question ici des sentiments d'appartenance et d'intégration qui à la longue se retrouvent chez tous, qu'ils soient nés au Maroc ou nés en France.

À la question "Manger marocain pour toi, qu'estce que cela signifie ?", un jeune homme français d'origine marocaine a répondu : "C'est viscéral... tu sais, je suis français, je suis né en France, je me sens français, mais quand je me regarde dans la glace, je vois bien que je ne le suis pas tout à fait et qu'une part de moi est ailleurs... et la société me le renvoie aussi. Alors manger marocain, ça calme ce conflit. Quand je suis en crise, je cuisine et je mange, le bénéfice est immédiat" (Moasin, 29 ans, né en France). On retrouve dans ces propos la dualité que vivent beaucoup de personnes nées en France de parents étrangers. La nourriture devient une preuve de "marocanité" et permet d'assumer ce que l'on est ou tout du moins l'assignation que les autres renvoient dans certains contextes.

Oscillant entre ressemblance et différence, entre familier et étranger, les jeunes rencontrés nous parlent d'un sentiment de perte de repères mais surtout de double rejet ${ }^{15}$. Ni tout à fait français, ni tout à fait marocain, il s'agit alors de résoudre les tiraillements entre un soi actuel et un soi possible $^{16}$. Contrairement aux apprentissages linguistiques ou religieux qui peuvent parfois prendre du temps, les nourritures produisent des sentiments apportant des réponses qui peuvent être déployées de façon immédiate. Dans ce climat d'écartèlement entre rejet et appartenances 
multiples, les processus de construction identitaire conduisent à la recherche d'une certaine continuité de soi ${ }^{17}$ que nous nous proposons de nommer processus de construction d'un "soimangeant" lorsque la dimension alimentation s'y ajoute. L'identité s'organise alors autour de dynamiques pour lesquelles les nourritures, médiatrices d'origine, donnent un sens plus ou moins cohérent à la vie et offrent les ressources qui permettent de s'inventer différent ${ }^{18}$.

Toujours dans l'expérience de la dualité, il y a un manger familial correspondant le plus souvent à un style vécu et présenté comme marocain et un manger public, du dehors, avec des consommations identifiées comme françaises : "À la maison, ce n'était pas que de la cuisine marocaine, mais cétait souvent marocain ou 'd'influence marocaine', alors que dehors, au restaurant, au foot, à l'école ou avec les copains, on mangeait français: des burgers, des steaks-frites, des sandwichs, du fromage, du vin, de la ventrèche même... des trucs de Français, quoi !" (Jamel, 32 ans, né en France).

D'autres se sont découverts différents par cette dualité. Mangeant marocain tous les midi à la maison, un jeune homme nous a déclaré s'être senti privé d'une part de son identité française lorsqu'il s'est mis à fréquenter la cantine du lycée. Il y a découvert les cordons bleus, le fromage, le bœuf bourguignon et les carottes Vichy : "Des choses basiques que tout le monde mange, nous, on n'y a pas eu accès. J'ai été un peu jaloux de ça. Quand on construit notre identité de Franco-Marocain, on a aussi besoin de se rattacher à des choses, et l'alimentation, c'est vraiment caractéristique de notre identité et de notre culture de Français... Déjà que l'on ne mange pas de porc et que l'on ne boit pas de vin, alors si en plus on nous enlève les quenelles... c'est dur de se dire que l’on est Français!" (Youssef, 29 ans, né en France).

Cette façon de prendre position par l'alimentation, nous la retrouvons aussi chez des personnes nées et socialisées au Maroc. Elles oscillent entre ici et là-bas en fonction des contextes d'interaction au sein desquels nous avons eu parfois une influence à aucun moment négligée. Intervient alors un jeu de mise en scène de soi: "Moi, je mange français, je suis comme toi, je suis comme tout le monde, je fais comme tout le monde, je suis en France alors je mange français" (Amhed, 58 ans, né au Maroc et arrivé en France au début des années 2000).

Pour les personnes nées au Maroc, la (re)socialisation alimentaire consiste en la mise en place de stratégies d'adaptation concrètes - comme nous l'avons vu plus haut - et de stratégies d'adaptation symbolique où l'expérience de la dualité force parfois à des ruptures: "Je pense sincèrement que ma façon de manger a contribué à mon intégration et à construire mon sentiment d'appartenance à la France. Quand jai débarqué du Maroc à Tarbes, à la fin des années 1970, j'ai tout de suite mangé comme tout le monde. C'est par l'alimentation que je suis entré en contact avec ce pays. Sans manger tu meurs, non ? Alors je me suis débrouillé et j'aisurtoutcassémescadres", (Mohammed, 60 ans, né au Maroc).

Pour "casser ses cadres" Mohammed est passé par L'alimentation sert d'ancrage dans un territoire et entre d'une façon particulière dans la construction des sentiments d'appartenance et d'intégration au groupe dans le contexte français. une phase troublante de déconstruction de tout un univers de croyances, normes et représentations liées à la dimension religieuse qui habite le modèle alimentaire marocain. Depuis, nourritures françaises et marocaines cohabitent dans sa façon de manger mais ne se rencontrent jamais: "Je ne mélange jamais les recettes. Les recettes françaises restent françaises et les recettes marocaines restent marocaines. Chez moi, il n'y a pas de mélange possible. Je ne vais pas non plus finir un repas marocain comme un couscous par du fromage." L'alimentation sert d'ancrage dans un territoire et entre d'une façon particulière dans la construction des sentiments d'appartenance et d'intégration au groupe dans le contexte français. 


\section{Un processus de mise en mémoire}

Même si certaines personnes rencontrées disent s'être intégrées selon le schéma assimilationniste, en matière d'alimentation, l'oubli de soi n'est jamais total. C'est ce que nous nommons "phénomènes de mise en mémoire". Ils se lisent dans certains récits de vie et montrent que même si l'on pense qu'il ne reste rien du souvenir d'un "soi-mangeant" marocain d'avant la migration, même si l'on a voulu marquer une nette "rupture $e^{19}$ " avec la socialisation alimentaire se rattachant à un "noyau dur ${ }^{20 "}$ expérimenté là-bas ou ici, même si pour certains

il devient possible d'oublier

L'alimentation intervient

comme une ressource,

pour entretenir la mémoire

à travers les habitudes alimentaires, les manières de manger, les techniques culinaires conservées, parfois avec les "bons" ingrédients, parfois avec de nouveaux produits, mais aussi une ressource pour explorer et faire se rencontrer les univers culturels. son passé et même si parfois l'on a oublié sa langue ou les façons de se mouvoir et d'"être", la mémoire sensorielle, elle, perdure et n'a pas besoin de cadres sociaux "pour réactiver un vécu de soi ancien ${ }^{21 "}$.

Ces phénomènes de mise en mémoire peuvent apparaître à tout âge et sont majoritairement réactivés dans des contextes particuliers. L'éloignement de la famille ou l'isolement du groupe de référence, par exemple: "Pendant longtemps j'ai mangé du porc, toute mon adolescence en fait, pour faire comme les copains et pour ne pas me sentir différent. Depuis que je vis ici à Toulouse, je suis retourné vers une alimentation plus 'traditionnelle" (Jamel, 32 ans, né en France). Le décès d'un proche : "C'est à la mort de mon grand-père au bled que j'ai demandé à ma grand-mère de m'apprendre quelques recettes, ça m'a touché... j’en ai eu besoin" (Younane, 29 ans, né en France). Un mariage où la cohabitation avec l'être aimé : "Depuis que je suis en couple je prends le temps de cuisiner des bons petits plats de chez nous le week-end, ce que je ne faisais jamais avant" (Samira, 30 ans, née en France).

La naissance d'un enfant, la vieillesse ou tout simplement une maladie qui, en mettant l'homme face à la mort, le fait se retourner sur son histoire: "Avant, je mangeais n'importe comment. Je mangeais surtout des choses françaises, en sauce, grasses, prises sur le pouce pendant mes déplacements professionnels. J'ai fait un infarctus il y a quelques années. Depuis je fais attention. Alors c'est vrai qu'on mange plus souvent marocain à la maison depuis" (Mohammed, 60 ans, né au Maroc).

Mémoire et identité sont au service l'une de l'autre, elles "se fécondent mutuellement, se fondent et se refondent pour produire une trajectoire de vie, une histoire, un mythe, un récit ${ }^{22}$. Le travail de mémoire est un opérateur majeur de la construction identitaire ; il s'agit d'un travail de réappropriation et de négociation que chacun opère vis-à-vis de son passé pour élaborer et nourrir son individualité. Ce processus se déploie dans un environnement où "lautre", les "autres"23", sont aussi acteurs et producteurs de sens et d'histoires en interaction avec celle de l'individu. Cette réalité invalide en grande partie l'approche essentialiste ou substantialiste de l'identité pour inviter à prendre en compte la relation que l'homme entretient avec le monde qui l'entoure et les bricolages qu'il met en œuvre pour se positionner sur le plan identitaire et maintenir le fil : comme Jamel (32 ans, né en France), plusieurs jeunes rencontrés ont déclaré avoir mangé haram (illicite) à un certain moment de leur adolescence - pour être ou faire comme les copains - puis sont retournés au halal (licite) plus tard - pour revenir aux traditions selon leurs mots ou se retrouver. L'individuel et le collectif se nourrissent aussi 
mutuellement. En mangeant, l'individu adopte les valeurs et les codes de son groupe d'appartenance, en même temps qu'il marque son individualité, parfois en s'adaptant, parfois en s'opposant, mais bien souvent en influençant en retour le collectif.

C'est dans ces "tricotages ${ }^{24 "}$ entre le collectif et le personnel, entre socialité et sociabilitée ${ }^{25}$, que s'inscrivent les processus identitaires et mémoriels pour lesquels l'alimentation intervient comme une ressource, pour entretenir la mémoire à travers les habitudes alimentaires, les manières de manger, les techniques culinaires conservées, parfois avec les "bons" ingrédients, parfois avec de nouveaux produits, mais aussi une ressource pour explorer et faire se rencontrer les univers culturels. Chez toutes les personnes rencontrées, nous avons relevé la présence d'objets totems, comestibles ou non, tantôt exposés dans les cuisines, tantôt cachés au fond des placards ${ }^{26}$. Symboles d'une fidélité aux origines, ces ingrédients, ces ustensiles de cuisine ou objets de la table ont été amenés du Maroc par eux-mêmes ou par les parents. L'entretien et la création du lien passent aussi par ces objets ; ils impliquent un désir de continuité et d'intégration à l'histoire d'un groupe. Ils peuvent aussi être oubliés, délaissés ou abandonnés; dans ce cas, loin de marquer une volonté de rupture, ils traduisent le désir d'aller vers l'autre et de le découvrir.

\section{La construction de deux univers}

Les données que nous avons recueillies délimitent clairement une séparation entre nourriture marocaine et nourriture française chez toutes les personnes rencontrées, qu'elles soient nées en France ou au Maroc. Même si des processus de métissage sont identifiables de toute part, notre corpus montre comment les interviewés créent l'univers "à la marocaine" et celui "à la française".
L'appellation des mets marque une frontière dans la construction de la similitude et de la différence : ils mangent des crêpes françaises pour le goûter et un hachis-kefta (hachis-parmentier dans lequel on aura ajouté des épices) pour le dîner. Chez Ibti (33 ans, née en France) le dimanche, c'est poulet grillé "à la marocaine".

Lorsqu'elle nous détaille la recette, on se rend compte qu'il ne s'agit finalement que d'un poulet rôti au four que Chez toutes les personnes rencontrées, mais plus fortement chez les enquêtés nés et socialisés en France, nous avons observé l'expérience d'une dualité entre manger marocain et manger français. l'on aura badigeonné d'une marinade faite d'un mélange d'ail écrasé, de paprika, de jus de citron et d'huile d'olive. Vu sous cet angle, c'est un plat qui, somme toute, fait partie aussi du manger français et que l'on retrouve ailleurs à travers le monde comme au Portugal. Sur quel critère vient alors se fonder l'appellation "à la marocaine" ? Sur un contexte, car la consommation se déroule en famille, entre Marocains, ou sur l'utilisation d'ingrédients tels que le paprika (poivron rouge non piquant) et l'huile d'olive, aliments totems au Maroc? Cette façon de se vivre et de manger marocain n'est pas tant liée à une recette quà des contextes d'interaction, à des événements. C'est dans ces moments de rencontre que la mémoire alimentaire se forge et permet d'entretenir le lien avec une histoire familiale.

C'est aussi autour de cette interprétation de ce qui est "français" et de ce qui est "marocain" que s'organisent les positionnements identitaires et les jeux d'appropriation alimentaire. Ce phénomène ne s'observe pas seulement chez des individus nés et socialisés en France, ni seulement chez les Marocains nés au Maroc, mais aussi chez des Français ou d'autres personnes encore. Cela fonctionne dans les deux sens. Pendant le ramadan, il se vend sur le marché d'Empalot à Toulouse des "crêpes mille trous", "baghrir" de leur vrai nom. 


\section{Conclusion}

Les échanges avec les femmes et les hommes marocains ou d'origine marocaine que nous avons rencontrés entre 2010 et 2013 permettent de comprendre comment, en situation d'immigration ou d'exil, les nourritures servent la construction sociale et l'entretien de leur identité et de leur mémoire, devenant par là même une ressource pour se positionner entre pays d'origine et société d'accueil. Leurs récits de vie montrent que, même si l'on pense qu'il ne reste rien de la mémoire, on n'oublie pas les odeurs et les goûts. LC'est dans cette optique que nous nous sommes attachées à recueillir des ressentis et à capter des univers de signification liés à l'alimentation et aux expressions identitaires.

Le processus de resocialisation alimentaire en trois temps que nous observons chez les individus nés et socialisés au Maroc révèle un parcours allant d'une phase de perturbation identitaire ("je suis perdu"), à un temps de (re)composition ("j'apprends", "je reconstruis") puis se termine par un processus d'appropriation (“je joue”, “je jongle avec"). Ces transformations sont à l'œuvre dans différents éléments des modèles alimentaires, tels que les dispositifs d'approvisionnement, les techniques culinaires ou encore les habitudes de consommation. Bien que présents chez toutes les personnes nées au Maroc que nous avons rencontrées, ces aspects sont plus clairement identifiables dans le parcours d'individus arrivés en France dans les années 1970-1980 qui, plus que les autres, ont dû réorganiser, à leur arrivée sur le territoire français, leur mode de vie et leur alimentation.

Les données que nous avons recueillies délimitent clairement une séparation entre nourriture marocaine et nourriture française. Même si, souvent, le manger marocain ne l'est plus tout à fait et que des processus de métissage sont identifiables de toutes parts, notre corpus montre comment les interviewés créent des univers "à la marocaine" ou "à la française". Dans ces dispositifs, différents supports tels que les livres de cuisine ou mobilier viennent renforcer la sémiosphère identitaire qui évolue au fil des contextes et des diverses rencontres autour des nourritures. En même temps qu'ils les renforcent, ces critères peuvent gommer les frontières entre les cultures alimentaires. On fait sien ou l'on rend dissemblable par différents moyens permettant de se positionner et de construire le sentiment d'appartenance à l'ici et/ ou au là-bas.

Chez toutes les personnes rencontrées, mais plus fortement chez les enquêtés nés et socialisés en France, nous avons observé l'expérience d'une dualité entre manger marocain et manger français. Il y a ce qui est le produit d'une mémoire familiale, identifié comme marocain, et ce qui est construit au présent dans lequel le manger français prend plus ou moins place aux côtés d'autres nourritures. Face à cette dualité, ces personnes semblent à la recherche d'une identité à trouver, à retrouver ou à créer.

Il s'agit pour eux de réguler une sorte de conflit intérieur en assumant ce qu'ils sont et qui fatalement se retrouve exposé dans l'espace public ou familial car on ne se cache pas pour manger. Les nourritures apportent des réponses visibles et immédiates dans l'expression identitaire vis-à-vis des deux groupes d'appartenance, le marocain et le français.

Dans ce jeu d'interactions entre assignation et auto-assignation, où l'individu se trouve confronté à une responsabilité d'intégration culturelle et sociale queles institutions traditionnelles semblent parfois ne plus assurer, à chacun de bricoler avec les outils à disposition pour trouver et exprimer sa place dans la société. Penser les symboliques des constructions identitaires et mémorielles met l'alimentation au cœur des problématiques de construction des sentiments d'appartenance et d'intégration. 